\title{
Lipid flippase dysfunction as a novel therapeutic target for endosomal anomalies in Alzheimer's disease
}

\author{
Authors \\ Nanaka Kaneshiro',2, Masato Komai ${ }^{1}$, Ryosuke Imaoka ${ }^{1}$, Atsuya Ikeda ${ }^{1}$, Yuji Kamikubo ${ }^{4}$ \\ Tadafumi Hashimoto ${ }^{3}$, Takeshi Iwatsubo ${ }^{3}$, Takashi Sakurai ${ }^{4}$, Takashi Uehara ${ }^{1}$, Nobumasa \\ Takasugi ${ }^{1,4,}{ }^{*}$
}

\section{Affiliation}

1. Department of Medicinal Pharmacology, Graduate School of Medicine, Dentistry, and Pharmaceutical Sciences, Okayama University, 1-1-1 Tsushima-naka, Kita-ku, Okayama 700-8530, Japan

2. Research Fellow of Japan Society for the Promotion of Science, Chiyoda-ku, Tokyo 102-0083, Japan.

3. Department of Neuropathology, Graduate School of Medicine, The University of Tokyo, 7-3-1 Hongo, Bunkyo-ku, Tokyo, 113-0033, Japan.

4. Department of Cellular and Molecular Pharmacology, Juntendo University Graduate School of Medicine, 2-1-1 Hongo, Bunkyo-ku, Tokyo 113-8421, Japan

* Correspondence should be addressed to - Nobumsa Takasugi, Ph.D., Department of Medicinal Pharmacology, Graduate School of Medicine, Dentistry and Pharmaceutical Sciences, Okayama University, Okayama 700-8530, Japan, email: ntakasu@okayamau.ac.jp

tel \& fax $+81-86-251-7985$

\begin{abstract}
$\beta$-amyloid precursor protein (APP) and their metabolites are deeply involved in the development of Alzheimer's disease (AD). Upon the upregulation of $\beta$-site APP cleaving enzyme 1 (BACE1), its product, the $\beta$-carboxyl-terminal fragment of APP ( $\beta C T F)$, is accumulated in the early stage of sporadic AD brains. $\beta C T F$ accumulation is currently considered the trigger for endosomal anomalies to form enlarged endosomes, one of the earliest pathologies in AD. However, the details of the underlying mechanism remain largely unclear. In this study, using BACE1 stably-overexpressing cells, we describe that lipid flippase subcomponent TMEM30A interacts with accumulated $\beta C T F$. Among the lipid flippases in endosomes, those composed of TMEM30A and active subcomponent ATP8A1 transports phospholipid, phosphatidylserine (PS), to the cytosolic side of the endosomes. The lipid flippase activity and cytosolic PS distribution are critical for membrane fission and vesicle transport. Intriguingly, accumulated $\beta C T F$ in model cells impaired lipid flippase physiological formation and activity, along with endosome enlargement. Moreover, in the brains of $A D$ model mice before the amyloid- $\beta(A \beta)$ deposition, the TMEM $30 A / \beta C T F$ complex formation occurred, followed by lipid flippase dysfunction. Importantly, our novel $A \beta / \beta C T F$ interacting TMEM30A-derived peptide "T-RAP" improved endosome enlargement and reduced $\beta C T F$ levels. These T-RAP effects could result from the recovery of lipid flippase activity. Therefore, we propose lipid flippase dysfunction as a key pathogenic event and a novel therapeutic target for AD.
\end{abstract}




\section{Introduction}

Amyloid- $\beta(A \beta)$ peptides are accumulated in the brains of patients with Alzheimer's disease $(A D)^{1}$ and are produced from the sequential cleavage of the $\beta$-amyloid precursor protein (APP) by the $\beta$-site APP cleaving enzyme 1 (BACE1) ${ }^{2,3,4}$ and $y$-secretase ${ }^{5}$. Although pieces of genetic and biological evidence suggest the link between $A \beta$ and $A D$ pathogenesis, several clinical trials based on the "amyloid hypothesis" have failed" ${ }^{6}$. Therefore, new ideas and therapeutic targets that complement the hypothesis are required for this field.

A recent report identified that traffic impairment, showing endosomal anomalies, is an early pathogenic event in $A D$ before $A \beta$ deposition ${ }^{7}$. In line with this, several studies in $A D$ model mice ${ }^{8}$, human iPSC-derived neurons ${ }^{9}$, and $A D$ brains ${ }^{10}$ have shown that the accumulated $\beta$-carboxyl-terminal fragment of APP ( $\beta C T F)$, the product of BACE1 and direct precursor of $A \beta$, is the cause of endosomal anomalies. Indeed, BACE1 expression and activity are upregulated at the early stage of sporadic $A D^{11,12}$. However, the details of the mechanism underlying the $\beta C T F$ mediated endosomal anomalies remain unclear.

Previously, we identified TMEM30A (CDC50A), a subcomponent of lipid flippases, as a candidate partner for $\beta C T F$. This complex mediates the formation of enlarged endosomes ${ }^{13}$. Most lipid flippases consist of TMEM30A and active subcomponents, P4-ATPases. These enzymes translocate phospholipids from the exoplasmic/luminal side to the cytoplasmic leaflet of the lipid bilayer to regulate phospholipids asymmetry ${ }^{14}$.

Phosphatidylserine (PS), one of the phospholipids, is a component of lipid bilayer and mainly localizes at the cytoplasmic leaflet ${ }^{14}$. PS level on the cytosolic side in endosomes determines the recruitment of PS-binding proteins to trigger membrane budding, which promotes subsequent vesicle fission and transport ${ }^{15,16}$. This PS asymmetry is regulated by one of the endosomal lipid flippases, those composed of TMEM30A and active subcomponent ATP8A1, which has a high affinity for PS ${ }^{14}$. These lines of evidence indicate that lipid flippase activity is essential for vesicular trafficking.

In this study, we investigated whether lipid flippase activity contributes to the $\beta C T F$ mediated endosomal anomalies and could be a novel therapeutic target for AD treatment.

\section{Results \\ BACE1 upregulation promotes complex formation between endogenous TMEM30A and accumulated $\beta C T F$, and endosomal anomalies}

To gain insight into the early pathology of $A D$, we established BACE1 stablyoverexpressing SH-SY5Y cell lines (SH-BACE1 cells). BACE1 produces $\beta 1$ or $\beta 11$ CTF depending on APP cleavage sites ${ }^{17}$. BACE1 upregulation increased the $\beta$-secretase products sAPP $\beta, \beta C T F$, and $A \beta$, although it severely reduced $\alpha$-secretase-cleaved carboxylterminal fragment ( $\alpha \mathrm{CTF})$, in good agreement with previous reports ${ }^{18}$ (Fig. $1 \mathrm{~A}$ and Supplementary Fig. 1A). We previously demonstrated that TMEM30A is a candidate partner for $\beta C T F$ ( $\beta 1 / \beta 11 C T F)$-mediated endosomal anomalies ${ }^{13}$. Supportively, CFP-TMEM30A, SC100 ${ }^{19}$, and SC89 (artificial $\beta 1$ and $\beta 11 \mathrm{CTF)} \mathrm{co-transfection} \mathrm{revealed} \mathrm{that}$ TMEM $30 A$ could interact with these $\beta C T F$ (Supplementary Fig. 1B). Although $\beta C T F$ in nontreated SH-BACE1 cells might deserve further analysis, we found that endogenous $\beta C T F$ exhibited resistance to mild detergents such as CHAPS (Supplementary Fig. 1C and D). Indeed, $\beta C T F$ insolubility in mild detergents was reported elsewhere ${ }^{20}$. Our result suggests that the environment surrounding $\beta C T F$ is particularly unique. Therefore, to verify whether endogenous TMEM30A interacts with accumulated $\beta$ CTF in SH-BACE1 cells, we treated $\gamma^{-}$ secretase inhibitor DAPT to accumulate APP-CTF, then performed co-immunoprecipitation analysis. In line with our previous findings, we observed an interaction between the endogenous TMEM $30 A$ and accumulated $\beta C T F$, but not $\alpha C T F$, and the BACE1 inhibitor cotreatment abolished this interaction (Fig. 1B).

Next, a stepwise iodixanol gradient organelle fractionation was performed as described previously ${ }^{21}$ to analyze the protein distributions. The distribution of LAMP2 (lysosome marker) and calreticulin (ER marker) showed no obvious change between $\mathrm{SH}$-control and SH-BACE1 cells (Supplementary Fig. 2A). In contrast, Rab5A, an early endosome marker, 
was distributed in fractions (Fr) 7 12 in SH-control cells (Fig. 1C) and its distribution was broadened to the heavier fractions (Fr 14 16) in SH-BACE1 cells. Similarly, $\beta C T F$, enriched in $\mathrm{Fr} 6 \sim 9$ in $\mathrm{SH}$-control cells, changed its distribution in the heavier fractions (Fr 14 16) in SH-BACE1 cells (Fig. 1C). Concomitantly, BACE1 was distributed in Fr 14 16 in SH-BACE1 cells (Supplementary Fig. 2A). Intriguingly, TMEM30A was mainly distributed in Fr 7 12 in $\mathrm{SH}$-control cells. However, in SH-BACE1 cells, its distribution drastically changed to Fr 14 16 where Rab5, $\beta C$ TF, and BACE1 abnormally co-localized (Fig. 1C). We observed no alteration in the TMEM30A and Rab5A protein levels between SH-control and SH-BACE1 cells (Fig. 1A and Supplementary Fig. 2B).

Next, we performed immunofluorescence analysis using BACE1 stably-overexpressing COS-7 cells (COS-BACE1), well-characterized in organelle morphology observations ${ }^{13,22,23}$. In contrast to the lack of alteration in the Rab5A protein level (Supplementary Fig. 2C), the mean of the Rab5A-positive puncta area and the frequency of size distribution $\left(\geq 0.1 \mu \mathrm{m}^{2}\right)$ were significantly increased in COS-BACE1 cells (Fig. 1D, E, and Supplementary Fig. 2D).

Our findings suggest that BACE1 increases $\beta C T F$ level, which interacts with TMEM $30 A$ and mediates endosomal anomalies.

\section{$\beta C T F$ accumulation triggers the lipid flippase activity impairment}

TMEM30A interacts with active subunits, P4-ATPases, to form lipid flippase, and contributes to stability, distribution, and activity of P4-ATPases ${ }^{14,24}$. Therefore, we hypothesized that lipid flippase activity is associated with TMEM30A/BCTF-mediated endosomal anomalies.

Immunoprecipitation analysis in the membrane fractions revealed that the physiological complex formation between TMEM30A and ATP8A1, an endosomal P4-ATPase which has a high affinity for PS, significantly decreased in SH-BACE1 cells. The BACE1 inhibitor treatment recovered this interaction in SH-BACE1 cells (Fig. 2A, B, and Supplementary Fig. 3A), indicating that this deficit is BACE1 activitydependent.

Since it is difficult to evaluate lipid flippase activity in organelles such as endosomes, we attempted to exploit the endosomal PS binding property of Evectin2, a promoting factor of membrane fission in vesicle transport to analyze the lipid flippase activity. Evectin-2 has a PS-specific pleckstrin-homology (PH) lipid-binding domain, and its association with the endosomal membrane depends on lipid flippase activity ${ }^{23,25}$. As previously reported ${ }^{23}$, the predominant distribution of Evectin-2 in the membrane fractions was abolished by TMEM30A knockdown (Fig. 2C, D, and Supplementary Fig. 3B). Intriguingly, Evectin-2 dissociated from the membrane fractions in SH-BACE1 cells and got redistributed upon the BACE1 inhibitor treatment without altering total Evectin-2 level (Fig. 2E and F).

To further quantify Evectin-2 localization in the endosomes, we used a NanoBiT luciferase reconstitution system. Large BiT ( $\mathrm{LgBiT})$ and Small BiT (SmBiT) are the parts of the Oplophorus gracilirostris-derived Nanoluc luciferase. As SmBiT has a low affinity $(\mathrm{Kd}=190 \mu \mathrm{M})$ to the $\mathrm{LgBiT}$, their interaction is fragile and reversible, and the luciferase activity is not reconstituted without their forced proximity by fused proteins ${ }^{26}$. We fused these subunits to Rab5A (LgBiT-Rab5) and Evectin-2 (SmBiT-Evectin-2) to monitor the Evectin-2 localization in the endosomes (Fig. 3A). Immunofluorescence analysis showed that LgBiTRab5 localized in the endosomes (Supplementary Fig. 4A). Moreover, similar to our biochemical analysis (Fig. 2C and D), TMEM30A knockdown significantly decreased the luciferase activity (Fig. 3B, C and Supplementary Fig. 4B). For further validation, we investigated the effect of Evectin-2 mutant on the PH domain (K20E), lacking the binding affinity to $\mathrm{PS}^{23}$. We observed significantly reduced luciferase activity in the K20E mutant (Supplementary Fig. 4C and D). These results clearly show that our reporter system could be used for semi-quantitative estimation of lipid flippase activity in endosomes.

Next, we applied this system to SH-BACE1 cells. Intriguingly, we observed a significant reduction in the lipid flippase activity, rescued by the BACE1 inhibitor treatment (Fig. 3D and E). To confirm that the BACE1-mediated APP cleavage is a prerequisite for this event, we 
performed APP knockdown in SH-BACE1 cells and detected lipid flippase activity recovery (Fig. 3F, G and Supplementary Fig. 5A-C). Interestingly, the APP knockdown failed to affect the lipid flippase activity in SH-control cells (Supplementary Fig. 5D). Furthermore, lipid flippase activity significantly decreased in SC100 stably overexpressing SH-SY5Y cells (Fig. $3 \mathrm{H}$ and $\mathrm{I}$ ).

Our data strongly support the hypothesis that $\beta$ CTF accumulation leads to lipid flippase dysfunction via abnormal complex formation with TMEM30A.

\section{TMEM30A/ $\beta C T F$ complex formation and subsequent lipid flippase dysfunction precede $A \beta$ deposition in $A D$ model mice}

Next, we explored the TMEM30A/ $\beta C T F$ complex formation and lipid flippase dysfunction at the early stage in $A D$ model mice. As $A 7$ model mice show a relatively slow $A \beta$ deposition, starting at approximately 9 months of age, we used these AD model mice as an appropriate model to observe the precursory phenomenon ${ }^{27}$. At 3 and 6 months, the ATP8A1, TMEM30A, and Evectin-2 protein levels were not significantly different between the WT and transgenic (Tg) mice (Supplementary Fig. 6A and B). Moreover, we observed no significant difference in the aCTF and $\beta C T F$ levels between the 3 - and 6-month-old $\mathrm{Tg}$ mice (Supplementary Fig. 6A and B). Intriguingly, TMEM30A interacted with $\beta C T F$ in both 3 - and 6 -month-old model mice (Fig. $6 \mathrm{~A}$ and $\mathrm{B}$ ). However, TMEM30A failed to interact with $A \beta$ oligomers, previously described as another factor in vesicular traffic impairment ${ }^{28}$, and with $A \beta$ monomers (Supplementary Fig. 7). Importantly, both lipid flippase formation (Fig. 4A and C) and Evectin-2 localization in the membrane fractions (Fig. 4A and D) significantly decreased in 6-month-old A7 mice. We confirmed that the indicated band is Evectin-2 (Supplementary Fig. 6C). Our results suggest that the TMEM30A/ $\beta C T F$ complex induces lipid flippase dysfunction, which precedes $A \beta$ deposition.

\section{$\beta C T F / A \beta$ interactive peptide "T-RAP" improves endosome enlargement}

We expected that the inhibition of the interaction between TMEM30A and $\beta C T F$ could improve lipid flippase dysfunction and endosomal anomalies. Previously, we identified that the extracellular domain of TMEM30A (TmEx) interacts with the $A \beta$ sequence of $\beta C T F^{13}$. We explored the interacting domain by sequential deletion of TmEx using GST-pulldown assay (Fig. 5A-C) and found that the 117-166 AA region contains the critical residues for the interaction with $\beta$ CTF (Fig. 5B-D). Using RaptorX prediction (http://raptorx.uchicago.edu/), we noticed that the conformation of the $\alpha$-helices and $\beta$-sheet exists in the well-conserved 125-150 AA region. We named this sequence "T-RAP" (Tmem30A related amyloidbeta interacting peptide) (Fig. 5A). We verified that GST-fused T-RAP efficiently pulled down $\beta C T F$ and $A \beta$, but not $\alpha C T F$ (Fig. 5B, D and Supplementary Fig. 8), indicating T-RAP has a high affinity for $A \beta N$-terminal sequence.

To assess whether the synthetic T-RAP peptide influences lipid flippase activity, we treated SH-SY5Y cells with T-RAP and performed a flippase activity assay. In advance, we confirmed that T-RAP displayed no toxicity at the density used in this study (Supplementary Fig. 9A). Although not significant, T-RAP showed a trend to improve lipid flippase activity in SH-BACE1 cells (Fig. 6 A and B). On the other hand, T-RAP $(50 \mu \mathrm{M})$ fused with the Trans-Activator of Transcription Protein (TAT) which is easily introduced into cells significantly rescued the lipid flippase activity in SH-BACE1 cells (Supplementary Fig. 9B). We consider that TAT-T-RAP could penetrate membranes more efficiently than T-RAP.

As lipid flippase dysfunction is $\beta C T F$ level-dependent, we investigated the involvement of T-RAP in APP metabolism. After a $48 \mathrm{~h}$ T-RAP peptide treatment, the sAPP $\beta$ and $\beta C T F$ levels significantly decreased without altering the full-length APP and TMEM30A protein levels (Fig. 6C and D). Next, we analyzed the T-RAP effect on endosomal morphology in COS-7 cells. Importantly, T-RAP significantly decreased the mean of the Rab5A-positive puncta area in COS-BACE1 cells without altering the total Rab5A protein level (Fig. 6E, F, and Supplementary Fig. 9C). These lines of evidence explain that T-RAP peptide improves endosomal anomalies by rescuing the lipid flippase activity. 


\section{Discussion}

$\beta C T F$-mediated endosomal anomalies in forming enlarged endosomes are considered early $A D$ pathogenic events ${ }^{10}$. In this study, we showed that lipid flippase activity in endosomes decreased by elevated $\beta C T F$ level and it contributes to endosomal anomalies. Moreover, in the $A D$ model mice brain, age-dependent lipid flippase dysfunction occurs before $A \beta$ deposition, supporting their strong link with the early pathogenic event. Importantly, we found that a novel $\beta C T F / A \beta$-interacting peptide "T-RAP" could recover the lipid flippase activity and endosomal anomalies. Therefore, our findings suggest that lipid flippase impairment is a driving mechanism of $\beta C T F$-mediated endosomal anomalies and present a novel therapeutic strategy for $A D$ treatment.

Endosomal anomalies are the signature for vesicular traffic impairment and could be found in the early phase of $A D$ and Down syndrome (DS) prior to $A \beta$ deposition ${ }^{7}$. As DS displays trisomy on chromosome 21, upregulating APP expression, many studies have focused on APP metabolites toxicity. Among these metabolites, the BACE1 product $\beta C T F$ is considered the driver for endosomal anomalies. Supportively, $\beta C T F$ accumulation is observed in $A D$ brains ${ }^{10}$, concomitant with upregulated BACE1 expression and activity in $A D$ brains $^{12}$. Several studies using human APP/Presenilin-1 familial AD mutant knock-in iPSCderived neurons ${ }^{9}$ or $3 \times \mathrm{Tg}-\mathrm{AD}$ model mice ${ }^{8}$ have shown that endosome enlargement depends on elevated $\beta C T F$ but not A $\beta$. Moreover, analysis of DS fibroblasts or Ts65Dn model mice has demonstrated that accumulated $\beta C T F$ induces endosome enlargement ${ }^{29}$. However, the underlying mechanisms are not fully explored.

To model the early pathogenic event of $A D$, we established a BACE1 stably overexpressing neuroblast cell line (SH-BACE1) and observed an increase in BACE1 products, $\beta 1 \mathrm{CTF}$ (C99) and $\beta 11 \mathrm{CTF}$ (C89) (Fig. 1A). Interestingly, we found that a subcomponent of lipid flippase, TMEM30A, is the interacting partner for these $\beta C T F$ (Fig. 1B and Supplementary Fig. 1B). Concomitantly, our organelle fractionation analysis showed a broadened distribution of the endosomal marker protein Rab5A to heavier fractions (Fr 14 16) in SH-BACE1 cells compared with normal endosome fractions (Fr 7 12) in control cells. Moreover, TMEM30A, $\beta C T F$, and BACE1 were co-distributed in these abnormal heavier fractions (Fig. 1C and Supplementary Fig. 2A). We hypothesize the vicious cycle, in which increased $\beta C T F$ triggers the interaction with TMEM30A to promote endosomal anomalies, and then the abnormal distribution of BACE1 further promotes $\beta C T F$ production. In connection with these results, immunofluorescence analysis using COS-7 cells showed the formation of enlarged endosomes in COS-BACE1 cells (Fig. 1D and E). Supportively, our previous study showed that $\beta C T F$ co-localized with TMEM30A in enlarged endosomes in COS-7 cells ${ }^{13}$. Another study proposed that intracellular $A \beta$, such as $A \beta$ oligomers, cause vesicular traffic impariment ${ }^{28}$. However, we failed to detect the interaction between TMEM $30 A$ and $A \beta$ monomers or oligomers in $A 7$ mice (Supplementary Fig. 7). Although we cannot fully rule out the possibility of the $A \beta$ oligomer involvement in a more advanced stage of $A D$, our data indicate that the major partner of TMEM30A, which mediated endosome enlargement, is $\beta C T F$.

There are two possible mechanisms underlying the TMEM30A/ $\beta C T F$ complex-mediated endosome enlargement. The first is lipid flippase dysfunction and the second is Rab5 overactivation.

Most lipid flippases consist of TMEM30A and active subcomponents, P4-ATPases ${ }^{30,31}$, and regulate phospholipid asymmetry in the lipid bilayer ${ }^{14}$. TMEM30A regulates proper cellular localization and activity of partners, P4-ATPases ${ }^{24,32}$. Intriguingly, we showed the complex formation of TMEM30A and ATP8A1, a brain-enriched endosomal P4-ATPase ${ }^{31}$, decreased in SH-BACE1 cells, and the inhibition of the BACE1 activity recovered the physiological complex formation (Fig. $2 \mathrm{~A}$ and $\mathrm{B}$ ). Our data suggest the hypothesis that upregulated BACE1 activity accumulates $\beta C T F$, which interacts with TMEM30A to interrupt the complex formation between TMEM30A and ATP8A1. Indeed, the age-dependent disruption of this lipid flippase formation was followed by TMEM30A/ $3 C T F$ complex formation 
in $A D$ model mice (Fig. 4A-C). We consider that the TMEM30A/BCTF complex further accumulates $\beta C T F$ in a vicious cycle, disrupting lipid flippase formation. Supporting this idea, TMEM30A overexpression in COS-7 cells accumulates $\beta C T F$, concomitantly with the complex formation of TMEM $30 A$ and $\beta$ CTF $^{13}$.

Endosomal lipid flippases transport phospholipids, like PS, to the cytosolic leaflet ${ }^{14}$. PS on the cytosolic side in endosomes recruits PS-binding proteins, such as Evectin-2, to promote membrane fission and trigger vesicle transport ${ }^{15,16}$. In this study, we focused on Evectin-2 distribution in the endosomes to indirectly monitor lipid flippase activity. We clarified that Evectin-2 distribution decreased depending on the increased $\beta C T F$ level in SH-BACE1 cells using biochemical analysis (Fig. 2) and the split-luciferase system (Fig. 3). These findings suggest that accumulated $\beta$ CTF decreases the lipid flippase activity in endosomes to reduce PS level on the cytosolic side. Moreover, the AD model mice analysis indicated decreased Evectin-2 distribution in the membrane fractions (Fig. 4A and D). Indeed, the ATP8A1 knockdown induces endosome-mediated membrane traffic defects ${ }^{22}$. Therefore, we propose the hypothesis that the TMEM30A/ $\beta$ CTF complex impairs lipid flippase formation and its activity to develop endosomal anomalies. It is important to note that our split-luciferase system can be applied to various organelle markers to estimate the localization of proteins and may be developed into a semi-quantitative method for measuring vesicular trafficking. Further validation is needed as a future study.

The second possible mechanism is Rab5 activation. Previous reports, using Rab5A constitutively active mutant (Q79L) ${ }^{33}$ and overexpressing mice ${ }^{34}$, showed Rab5 positive enlarged endosomes. Additionally, elevated $\beta C T F$ can form a complex with APPL1, a Rab5 effector protein, which mediates Rab5 activation and endosome enlargement in DS fibroblasts and $A D$ brains ${ }^{10}$. Consistent with these reports, we observed the upregulation of BACE1 mediated the abnormal co-distribution of Rab5A and BCTF to alter Rab5A-positive endosomal morphology (Fig. 1C-E). Therefore, accelerated $\beta C T F$ accumulation by its complex formation with TMEM30A might activate Rab5 to induce endosome enlargement. It would deserve further investigation that lipid flippase dysfunction contributes to Rab5 activation-dependent or independent endosomal anomalies.

Questions still remain concerning how lipid flippase-mediated endosomal anomalies could contribute to AD pathogenesis. Previously, APP-dependent endosomal anomalies and the traffic impairment of nerve growth factor (NGF), which resulted in neuronal atrophy, were observed in rodent neuron models ${ }^{29}$. Another study demonstrated that TMEM30A deficiency influenced SAPP $\beta$ and $\beta C T F$ levels, as well as $A \beta / p 3$ production via the increased $\beta / \alpha-$ secretase processing of APP ${ }^{35}$. Therefore, lipid flippase dysfunction possibly mediates APP metabolic changes or vesicle transport deficit such as NGF to contribute to $A D$ pathogenesis.

Since vesicular traffic impairment has been implicated as the contributor of AD pathology, $\beta C T F$-mediated endosomal anomalies might be promising therapeutic targets for treating AD. The BACE1 inhibitor might be a candidate. According to this notion, $\beta$-secretase inhibitor IV recovered lipid flippase function (Fig. 2E, F, and Fig. 3E). However, the study of BACE1 knockout mice showed unexpected neuronal phenotypes, such as schizophrenia endophenotypes or spine density reduction, originating from abrogated $\beta$-secretase processing of different substrates such as neuregulin 1 (NRG1) ${ }^{36,37}$. Further therapeutic candidates are pharmacological chaperons to stabilize retromers and limit APP processing in the endosomes by enhancing vesicle transport ${ }^{38}$. There is also a concern that the compound displays a too wide effect range and side effects ${ }^{39}$. These lines of evidence remind us to develop more specific therapeutic targets reflecting AD pathology.

We observed a progressive decline of lipid flippase function in $A D$ model mice (Fig. 4A and D). Moreover, a genome-wide association study identified AD risk variants in one of the P4-ATPases, ATP8B440. Therefore, lipid flippase dysfunction could be associated with $A D$ pathogenesis and the optimal target based on $A D$ pathology. Intriguingly, we identified a TMEM30A-derived peptide, "T-RAP," specifically interacting $\beta C T F$ and A $\beta$. TRAP trended to rescue the lipid flippase activity and improved endosome enlargement in BACE1 stably overexpressing cells (Fig. 6B, E, F, and Supplementary Fig. 9B). We 
hypothesize that T-RAP enfolds $\beta C T F$ to inhibit the interaction with TMEM30A, then recovers the lipid flippase physiological formation and activity. This functional lipid flippase recovery could prevent $\beta C$ TF-mediated endosomal anomalies, enhancing vesicle transport (Fig. 7). TRAP also decreased SAPP $\beta$ and $\beta C T F$ levels (Fig. $6 C$ and D), which means the peptide corrects vesicle transport to normalize APP processing. Another possibility is that T-RAP directly binds to the proximal site of BACE1 cleavage of APP. Moreover, T-RAP is hydrophilic and easy to handle biochemically. Although further T-RAP specificity analyses would be required, we propose that T-RAP and related molecules might be the optimal candidates for AD therapeutics.

Three main problems could be distinguished in $A D$ treatment from the perspective of vesicular traffic impairment. First, the details of the mechanisms underlying the endosomal anomalies were unclear. Second, the effective measurement of vesicular trafficking has not been established. Third, endosomal anomaly-related drug treatment has not yet been developed. In this study, we propose lipid flippase dysfunction as a mechanistic contributor

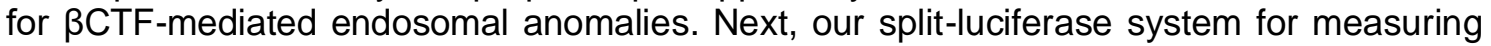
endosomal lipid flippase activity could be used for the development of quantitative vesicle transport measurements. Finally, we identified a novel therapeutic candidate for $\beta C T F$ mediated endosomal anomalies. Therefore, we present new insights into AD treatment for targeting the early pathogenesis, endosomal anomalies.

335

336

337

338

339

340

\section{Methods \\ Usage of mouse brains}

APP transgenic mice (A7 line) have been previously generated ${ }^{27}$ and genotyped using specific primers ${ }^{41}$. All mice were kept under specific pathogen-free conditions and fed a regular diet (Oriental Yeast). The animal care and use procedures were approved by the Institutional Animal Care and Use Committees of the University of Tokyo (18-P-108).

\section{Statistical analysis}

All experimental data are expressed as the mean \pm SEM. The experiments were analyzed with two-tailed Student's t-test or one-way ANOVA with Bonferroni's multiple comparisons test using the GraphPad Prism8 software. The statistical significance is indicated as follows: ${ }^{\star} P<0.05,{ }^{* \star} P<0.01,{ }^{* *} P<0.001,{ }^{* * \star *} P<0.0001$.

\section{Supplementary information}

Detailed information of the materials and methods, original western blot data of Fig. 1-5, as well as the supplementary figures are available in Supplementary information file.

\section{Acknowledgments}

We are grateful to Dr. T. Tomita (University of Tokyo), Dr. N. Nukina (Doshisha University) and Legend research grant in 2019 for providing research materials. This work was supported by the Grant-in-Aid for Scientific Research (C) from the Japan Society for the Promotion of Science (17K08272 and 20K07014), Research Grant from the Ryobi Teien Memorial Foundation, Life Science Foundation of Japan (NT), Grant-in-Aid for JSPS Fellows (NK).

\section{Author contributions}

N.K., and N.T., designed the research; N.K., R.I., M.K., A.I., and N.T. performed the experiments and analyzed data; T.H. and T.S. prepared animal samples and provided antibodies, respectively; N.K., and N.T., wrote the paper; Y.K., T.H., T.I., T.S., and T.U. provided advice and helped write the article. All authors read and approved the final manuscript.

\section{Conflict of interest}

369 The authors declare that they have no conflict of interest. 
370

\section{References}

1. Hardy, J. \& Selkoe, D. J. the Amyloid Hypothesis of Alzheimer 's Disease: Progress and Problems on the. Science 297, 353-6 (2002).

2. Vassar, R. et al. $\beta$-Secretase cleavage of Alzheimer's amyloid precursor protein by the transmembrane aspartic protease BACE. Science 286, 735-741 (1999).

3. Fairbanks, M. et al. Purification and cloning of amyloid precursor protein $\beta$-secretase from human brain. Nature 705, 537-540 (1999).

4. Yan, R. et al. Membrane-anchored aspartyl protease with Alzheimer's disease $\beta$ secretase activity. Cereb. Cortex 2558, 533-537 (1999).

5. Takasugi, N. et al. The role of presenilin cofactors in the $y$-secratase complex. Nature 422, 438-441 (2003).

6. Huang, L. K., Chao, S. P. \& Hu, C. J. Clinical trials of new drugs for Alzheimer disease. J. Biomed. Sci. 27, 1-13 (2020).

7. Cataldo, A. M. et al. Endocytic pathway abnormalities precede amyloid $\beta$ deposition in sporadic alzheimer's disease and down syndrome: Differential effects of APOE genotype and presenilin mutations. Am. J. Pathol. 157, 277-286 (2000).

8. Lauritzen, I. et al. The $\beta$-secretase-derived C-terminal fragment of $\beta A P P, C 99$, but not $A \beta$, is a key contributor to early intraneuronal lesions in triple-transgenic mouse hippocampus. J. Neurosci. 32, 16243-16255 (2012).

9. Kwart, D. et al. A Large Panel of Isogenic APP and PSEN1 Mutant Human iPSC Neurons Reveals Shared Endosomal Abnormalities Mediated by APP $\beta$-CTFs, Not A $\beta$. Neuron 104, 256-270.e5 (2019).

10. Kim, S. et al. Evidence that the rab5 effector APPL1 mediates APP- $\beta C T F$-induced dysfunction of endosomes in Down syndrome and Alzheimer's disease. Mol. Psychiatry 21, 707-716 (2016).

11. Zetterberg, $\mathrm{H}$. et al. Elevated cerebrospinal fluid BACE1 activity in incipient alzheimer disease. Arch. Neurol. 65, 1102-1107 (2008).

12. Ahmed, R. R. et al. BACE1 and BACE2 enzymatic activities in Alzheimer's disease. J. Neurochem. 112, 1045-1053 (2010).

13. Takasugi, N. et al. TMEM30A is a candidate interacting partner for the $\beta$-carboxylterminal fragment of amyloid- $\beta$ precursor protein in endosomes. PLoS One (2018).

14. Andersen, J. P. et al. P4-ATPases as Phospholipid Flippases-Structure, Function, and Enigmas. Front. Physiol. 7, 275 (2016).

15. Leventis, P. A. \& Grinstein, S. The distribution and function of phosphatidylserine in cellular membranes. Annual Review of Biophysics vol. 39 407-427 (2010).

16. Varga, K., Jiang, Z. J. \& Gong, L. W. Phosphatidylserine is critical for vesicle fission during clathrin-mediated endocytosis. J. Neurochem. 152, 48-60 (2020).

17. Deng, $Y$. et al. Amyloid- $\beta$ protein $(A \beta)$ Glu11 is the major $\beta$-secretase site of $\beta$-site amyloid- $\beta$ precursor protein-cleaving enzyme 1(BACE1), and shifting the cleavage site to A $\beta$ Asp1 contributes to Alzheimer pathogenesis. Eur. J. Neurosci. 37, 1962-1969 (2013).

18. Vetrivel, K. S. et al. Loss of cleavage at $\beta^{\prime}$-site contributes to apparent increase in $\beta$ amyloid peptide (A $\beta$ ) secretion by $\beta$-secretase (BACE1)-glycosylphosphatidylinositol (GPI) processing of amyloid precursor protein. J. Biol. Chem. 286, 26166-26177 (2011).

19. Iwata, H., Tomita, T., Maruyama, K. \& Iwatsubo, T. Subcellular Compartment and Molecular Subdomain of $\beta$-Amyloid Precursor Protein Relevant to the A 342 -promoting Effects of Alzheimer Mutant Presenilin 2. J. Biol. Chem. 276, 21678-21685 (2001).

20. Sakurai, T. et al. Membrane microdomain switching: A regulatory mechanism of amyloid precursor protein processing. J. Cell Biol. 183, 339-352 (2008).

21. Lee, M. S. et al. APP processing is regulated by cytoplasmic phosphorylation. J. Cell Biol. 163, 83-95 (2003).

22. Lee, S. et al. Transport through recycling endosomes requires EHD 1 recruitment by a phosphatidylserine translocase. EMBO J. 34, 669-688 (2015).

23. Matsudaira, T. et al. Endosomal phosphatidylserine is critical for the YAP signalling pathway in proliferating cells. Nat. Commun. 8, (2017).

24. Coleman, J. a \& Molday, R. S. Critical Role of the $\beta$-Subunit CDC50A in the Stable 
Expression, Assembly, Subcellular Localization, and Lipid Transport Activity of the P4ATPase ATP8A2. J. Biol. Chem. 286, 17205-17216 (2011).

25. Lee, S. et al. Impaired retrograde membrane traffic through endosomes in a mutant $\mathrm{CHO}$ cell defective in phosphatidylserine synthesis. Genes to Cells 17, 728-736 (2012).

26. Dixon, A. S. et al. NanoLuc Complementation Reporter Optimized for Accurate Measurement of Protein Interactions in Cells. ACS Chem. Biol. 11, 400-408 (2016).

27. Yamada, K. et al. $A \beta$ immunotherapy: Intracerebral sequestration of $A \beta$ by an anti-A $\beta$ monoclonal antibody 266 with high affinity to soluble A $\beta$. J. Neurosci. 29, 11393-11398 (2009).

28. Umeda, T. et al. Intracellular amyloid $\beta$ oligomers impair organelle transport and induce dendritic spine loss in primary neurons. Acta Neuropathol. Commun. 3, 51 (2015).

29. Xu, W. et al. Amyloid precursor protein-mediated endocytic pathway disruption induces axonal dysfunction and neurodegeneration. J. Clin. Invest. 126, 1815-1833 (2016).

30. Bryde, S. et al. CDC50 proteins are critical components of the human class-1 P 4ATPase transport machinery. J. Biol. Chem. 285, 40562-40572 (2010).

31. Wang, J. et al. Proteomic Analysis and Functional Characterization of P4-ATPase Phospholipid Flippases from Murine Tissues. Sci. Rep. 8, 10795 (2018).

32. Van Der Velden, L. M. et al. Heteromeric interactions required for abundance and subcellular localization of human CDC50 proteins and class 1 P4-ATPases. J. Biol. Chem. 285, 40088-40096 (2010).

33. Wegener, C. S. et al. Ultrastructural characterization of giant endosomes induced by GTPase-deficient Rab5. Histochem. Cell Biol. 133, 41-55 (2010).

34. Pensalfini, A. et al. Endosomal Dysfunction Induced by Directly Overactivating Rab5 Recapitulates Prodromal and Neurodegenerative Features of Alzheimer's Disease. Cell Rep. 33, 108420 (2020).

35. Tambini, M. D. \& D'Adamio, L. Loss of CDC50A function drives $A \beta / p 3$ production via increased $\beta / \alpha$-secretase processing of APP. bioRxiv (2020). doi:10.1101/2020.11.12.379636.

36. Vassar, R. BACE1 inhibitor drugs in clinical trials for Alzheimer's disease. Alzheimer's Res. Ther. 6, 1-14 (2014).

37. Savonenko, A. V et al. Alteration of BACE1-dependent NRG1/ErbB4 signaling and schizophrenia-like phenotypes in BACE1-null mice. 105, 10-15 (2008).

38. Mecozzi, V. J. et al. Pharmacological chaperones stabilize retromer to limit APP processing. Nat. Chem. Biol. 10, 443-449 (2014).

39. Berman, D. E., Ringe, D., Petsko, G. A. \& Small, S. A. The Use of Pharmacological Retromer Chaperones in Alzheimer's Disease and other Endosomal-related Disorders. Neurotherapeutics 12, 12-18 (2015).

40. Holstege, H., Hulsman, M., Charbonnier, C. \& Grenier-boley, B. Exome sequencing identifies novel AD-associated Abstract Background: With the development of nextgeneration sequencing technologies. medRxiv (2020). doi:10.1101/2020.07.22.20159251.

41. Hashimoto, T. et al. Collagenous Alzheimer amyloid plaque component impacts on the compaction of amyloid- $\beta$ plaques. Acta Neuropathol. Commun. 8, (2020). 
Figure 1. BACE1 upregulation induces the complex formation between TMEM30A and $\beta C T F$ and endosomal anomalies. (A) Immunoblotting analysis for APP metabolites and TMEM30A in SH-control and SH-BACE1 cells. $\beta$-secretase inhibitor IV $(10 \mu \mathrm{M})$ was treated to SH-BACE1 cells. (B) Co-immunoprecipitation analysis using TMEM30A antibody in the accumulation of $\beta C T F$ by the DAPT $(10 \mu \mathrm{M})$ treatment or co-treatment with $\beta$-secretase inhibitor IV $(10 \mu \mathrm{M})$ for $24 \mathrm{~h}$. (C) lodixanol gradient fractionation of the homogenates from SH-control and SH-BACE1 cells. For the endosome marker, Rab5A was used. (D) COScontrol and COS-BACE1 cells were immunostained for Rab5A (Red). DAPI stained the nucleus (Blue). Scale bars: $10 \mu \mathrm{m}$. Representative z-stack images were captured using a $60 x$ objective lens (zoom x2.6). (E) Quantitation of size distribution $\left(\geq 0.1 \mu \mathrm{m}^{2}\right)$ and mean area of Rab5A-positive puncta ( $n=3$, mean \pm SEM, two-tailed Student's t-test, ${ }^{\star} P<0.05$, $\left.{ }^{\star *} P<0.01\right)$.

Figure 2. BACE1 upregulation induces lipid flippase dysfunction depending on the BACE1 activity. (A) The membrane fractions from SH-control and SH-BACE1 cells applied to co-immunoprecipitation analysis using TMEM30A antibody. Cells were treated with the $\beta$ secretase inhibitor IV $(10 \mu \mathrm{M})$ for $48 \mathrm{~h}$. Calreticulin was used as a loading control of membrane fractions. (B) Quantification of ATP8A1 co-immunoprecipitated by TMEM30A antibody in Fig. $2 A$ ( $n=3$, mean \pm SEM, one-way ANOVA Bonferroni's multiple comparisons test, $\left.{ }^{*} P<0.05,{ }^{\star \star} P<0.01\right)$. (C) Immunoblotting analysis for Evectin-2 in total cell lysates and membrane fractions (MF) in the knockdown of TMEM30A in SH-SY5Y cells. (D) Quantification of Evectin-2 localization in whole cell lysates or MF ( $n=3$, mean \pm SEM, twotailed Student's t-test, $\left.{ }^{\star} P<0.05\right)$. (E) Immunoblotting analysis for Evectin-2 in total cell lysates and MF in the treatment of the $\beta$-secretase inhibitor IV $(10 \mu \mathrm{M})$ for $48 \mathrm{~h}$. (F) Quantification of the Evectin-2 localization in total lysates or MF $(n=3$, mean \pm SEM, one-way ANOVA Bonferroni's multiple comparisons test, $\left.{ }^{*} P<0.05,{ }^{* *} P<0.01\right)$.

Figure 3. The semi-quantitative analysis shows the lipid flippase activity in endosomes is decreased depending on the levels of $\beta$ CTFs. (A) Schematic view of the measurement methods for endosomal lipid flippase activity using a split-luciferase assay system. Luciferase subunits, $\mathrm{LgBiT}$ or SmBiT, were fused with Rab5A or Evectin-2, respectively. The reconstitution of luciferase activity depends on the endosomal distribution of Evectin-2, which reflects the endosomal lipid flippase activity (Fig.2). (B) Immunoblotting analysis for LgBiTRab5, SmBiT-Evectin-2, and TMEM30A using HA, FLAG, and TMEM30A antibodies in the knockdown of TMEM30A for $72 \mathrm{~h}$ in SH-SY5Y cells. (C) Quantification of the luciferase activity in the knockdown of TMEM30A for $72 \mathrm{~h}$ in SH-SY5Y cells $(n=3$, mean \pm SEM, twotailed Student's t-test, $\left.{ }^{*} P<0.05\right)$. (D) Immunoblotting analysis for LgBiT-Rab5, SmBiTEvectin-2, and APP-CTF in the treatment of $\beta$-secretase inhibitor IV $(10 \mu \mathrm{M})$ for $48 \mathrm{~h}$. (E) Quantification of the luciferase activity for treating $\beta$-secretase inhibitor IV $(10 \mu \mathrm{M})$ for $48 \mathrm{~h}$ $\left(n=3\right.$, mean \pm SEM, one-way ANOVA Bonferroni's multiple comparisons test, ${ }^{*} P<0.05$, $\left.{ }^{* \star \star \star} P<0.0001\right)$. (F) Immunoblotting analysis for LgBiT-Rab5, SmBiT-Evectin-2, APP, and APP-CTF in the knockdown of APP for $72 \mathrm{~h}$. (G) Quantification of the luciferase activity in the knockdown of APP for $72 \mathrm{~h}(n=4$, mean \pm SEM, one-way ANOVA Bonferroni's multiple comparisons test, $\left.{ }^{*} P<0.05\right)$. (H) Immunoblotting analysis for SC100, LgBiT-Rab5, and SmBiT-Evetin-2. P.C is the control for SC100 and aCTF. (I) Quantification of the luciferase activity in $\mathrm{SH}$-control and SH-SC100 cells $48 \mathrm{~h}$ after the transfection of luciferase subunits $\left(n=3\right.$, mean \pm SEM, two-tailed Student's t-test, $\left.{ }^{* *} P<0.01\right)$.

Figure 4. TMEM30A interacts with $\beta C T F$ in $A 7$ mice, which follows by lipid flippase dysfunction. (A) The membrane fractions from WT and A7 mice brain at 3-or 6-month-old 
applied to co-immunoprecipitation analysis using TMEM30A antibody. $\mathrm{Na}+/ \mathrm{K}+-\mathrm{ATP}$ ase was formation of (B) TMEM30A and $\beta$ CTF or (C) TMEM30A and ATP8A1 ( $n=3$, mean \pm SEM, two-tailed Student's t-test, $\left.{ }^{*} P<0.05,{ }^{* *} P<0.01\right)$. (D) Quantification of Evectin-2 localization in membrane fractions $\left(n=3\right.$, mean \pm SEM, two-tailed Student's t-test, $\left.{ }^{* *} P<0.01\right)$.

Figure 5. Identification of T-RAP peptide. (A) Schematic view of the sequential deletion constructs of GST-TmEx (Extracellular-domain of TMEM30A) and GST fused TMEM30A (117-166 AA) and "T-RAP" sequence. Lowest panel: The conservation of the T-RAP sequence among indicated species and predicted structure by Raptor-X. (B) Coomassie's brilliant blue staining of purified GST-fusion proteins in Fig 5A. (C, D) GST-pull down from HEK293 lysate transfected with SC100.

Figure 6. $\beta C T F$ interacting peptide 'T-RAP' shows a trend to rescue lipid flippase activity and improves the endosomal anomalies in BACE1 upregulation. (A) Immunoblotting analysis for SmBiT-Evectin-2 and LgBiT-Rab5 after the treatment of T-RAP $(10 \mu \mathrm{M})$ for $48 \mathrm{~h}$. (B) Quantification of the luciferase activity in T-RAP $(10 \mu \mathrm{M})$ treatment for $48 \mathrm{~h}$ ( $n=5$, mean \pm SEM, one-way ANOVA Bonferroni's multiple comparisons test). (C) Immunoblotting analysis for APP metabolites and TMEM30A in T-RAP $(10 \mu \mathrm{M})$ treatment for $48 \mathrm{~h}$. (D) Quantification of SAPP $\beta$ and $\beta C T F$ in Fig. 6C ( $n=3$, mean \pm SEM, one-way ANOVA Bonferroni's multiple comparisons test, ${ }^{*} P<0.05$, $\left.{ }^{* *} P<0.01,{ }^{* \star *} P<0.001\right)$. (E) COScontrol and COS-BACE1 cells were immunostained for Rab5A after T-RAP (10 $\mu \mathrm{M})$ treatment for $48 \mathrm{~h}$. Scale bars: $20 \mu \mathrm{m}$. Representative z-stack images were captured using a 60x objective lens. (F) Quantification of the mean Rab5A positive puncta area $(n=4$, mean \pm SEM, one-way ANOVA Bonferroni's multiple comparisons test, ${ }^{*} P<0.05,{ }^{*} P<0.01$ ).

Figure 7. Schematic view of the predicted mechanism of vesicular traffic impairment in $A D$, and therapeutic effect of T-RAP.

In $A D$, the complex formation between TMEM30A and accumulated $B C T F$ can interrupt lipid flippase physiological formation and its activity in endosomes, decreasing cytosolic PS levels required for membrane fission. Thus, the defect of membrane fission induces endosome enlargement and inhibits vesicle transport. On the other hand, the treatment of $\beta C T F$ interacting peptide, T-RAP, can prevent the TMEM30A/ $\beta C T F$ interaction, resulting in improved lipid flippase function and endosome enlargement. Therefore, T-RAP treatment can promote vesicle transport. 
bioRxiv preprint doi: https://doi.org/10.1101/2021.07 30.454423; this version posted August 29, 2021. The copyright holder for this preprint (which was not certified by peer review) is the author/funder, who has granted bioRxiv a license to display the preprint in perpetuity. It is made available under aCC-BY-NC-ND 4.0 International license.

Fig.1

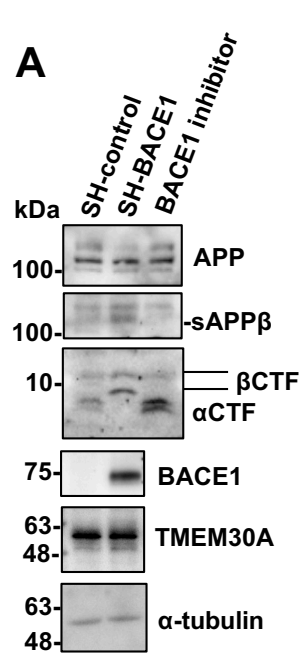

D

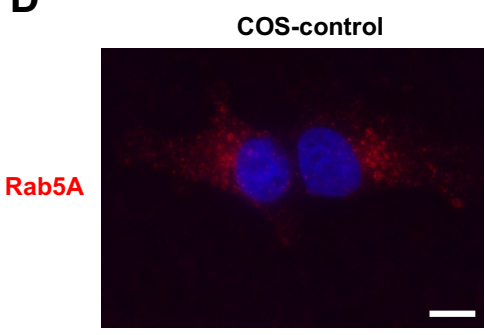

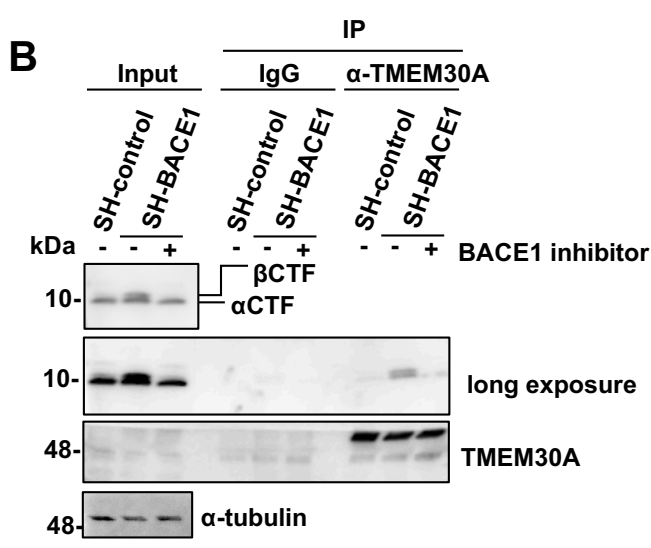

C

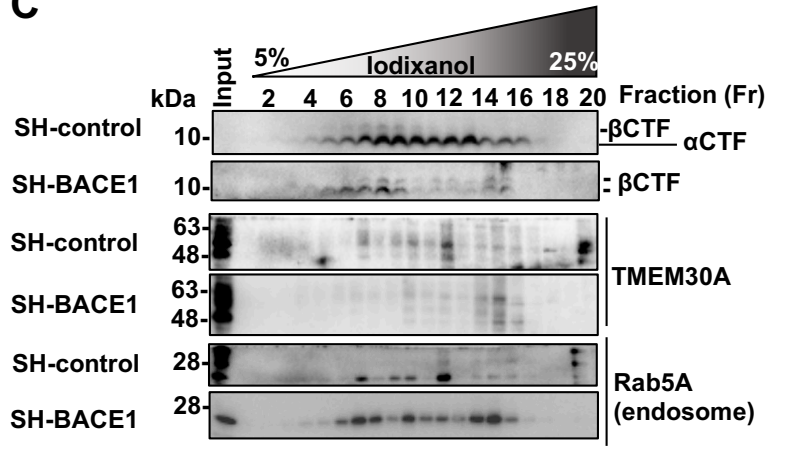

E

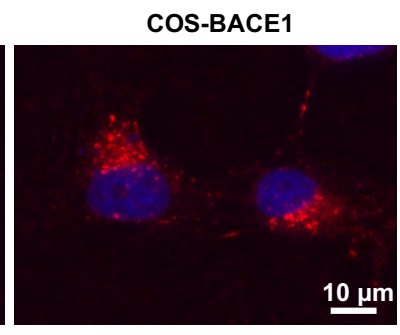

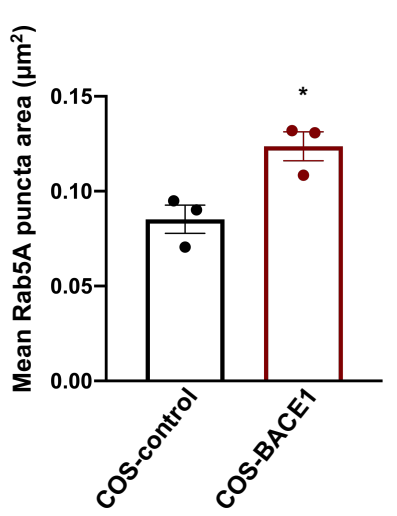

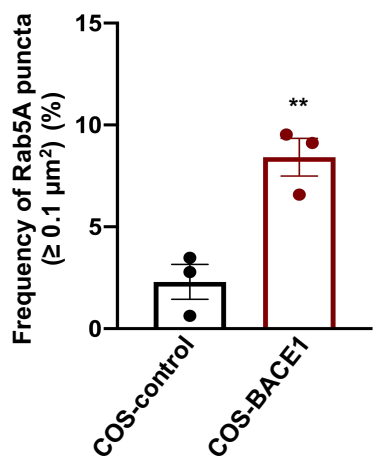




\section{Fig.2}

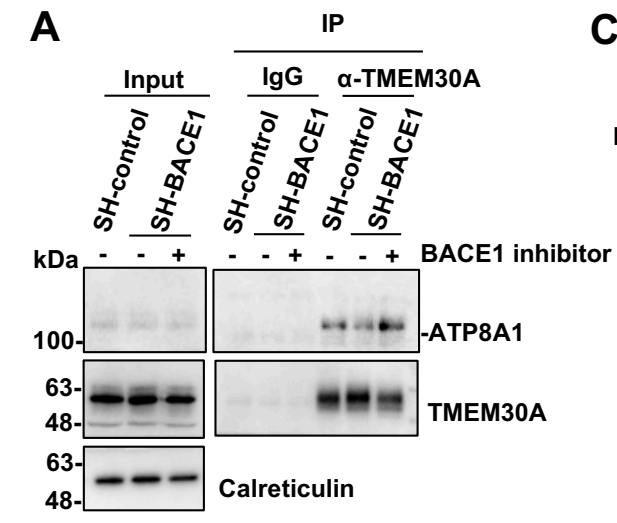

B

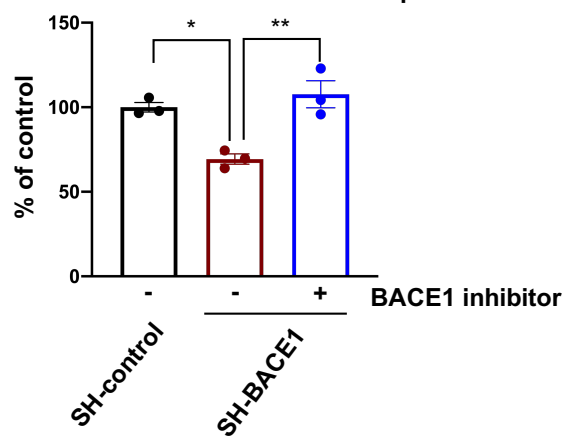

C
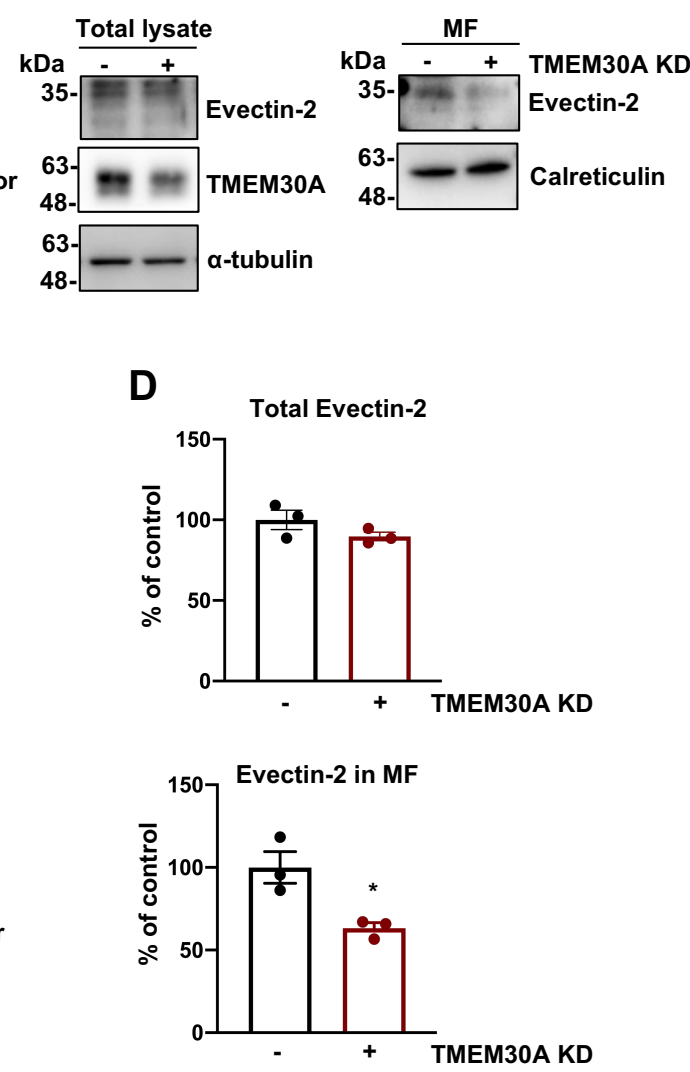

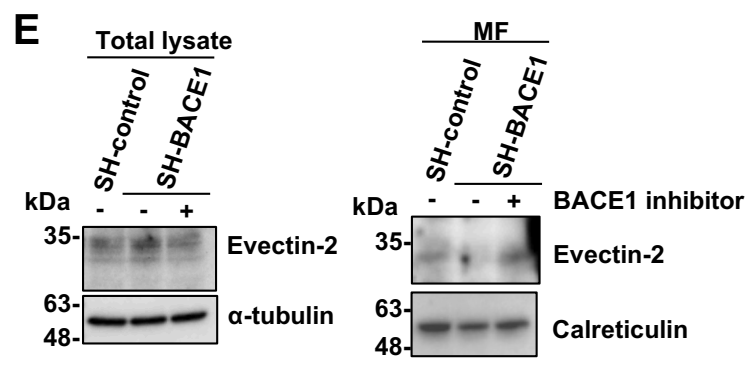

$\mathbf{F}$

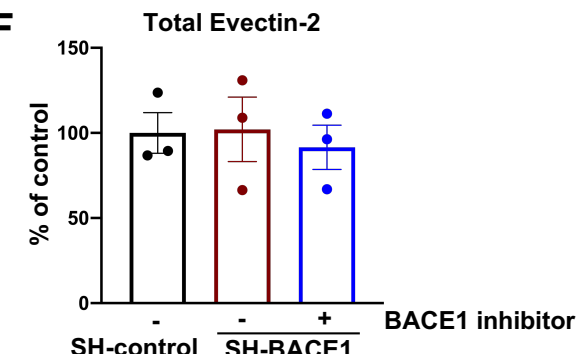
SH-control $\overline{\text { SH-BACE1 }}$

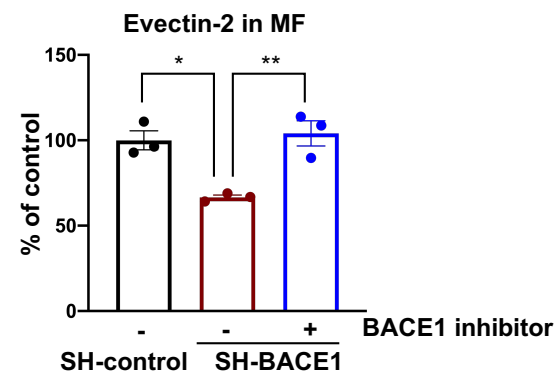




\section{Fig.3}

A

Scheme of split-luciferase system

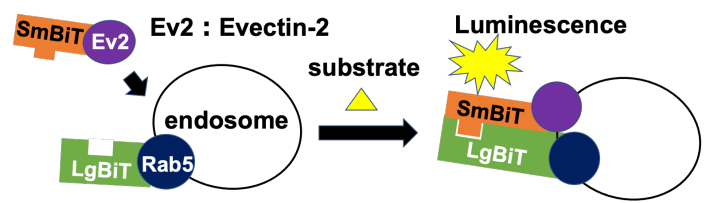

B

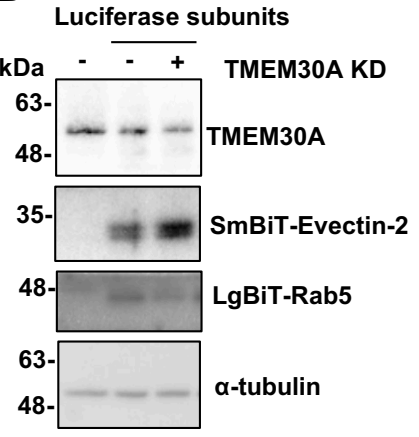

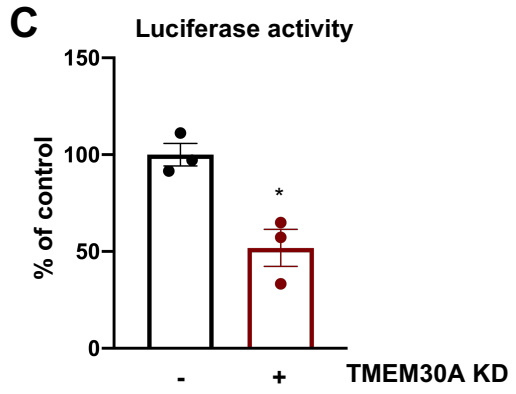

D

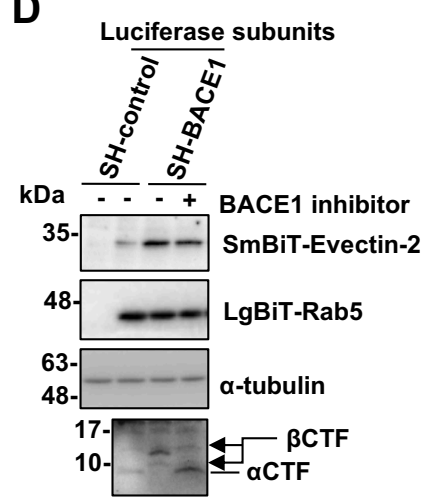

E

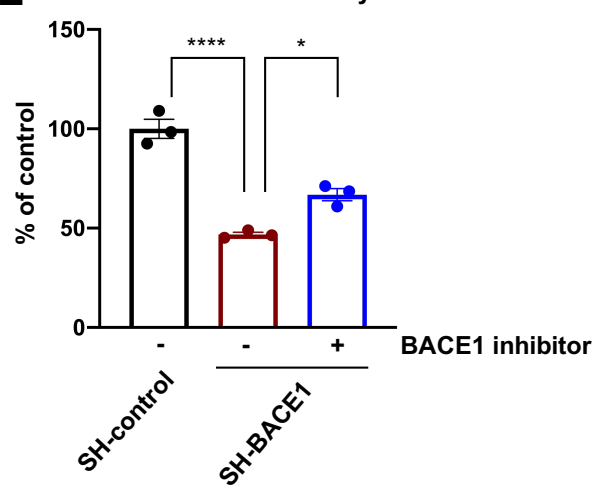

F Luciferase subunits

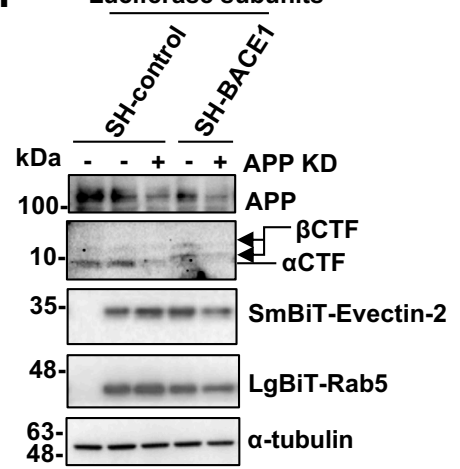

G

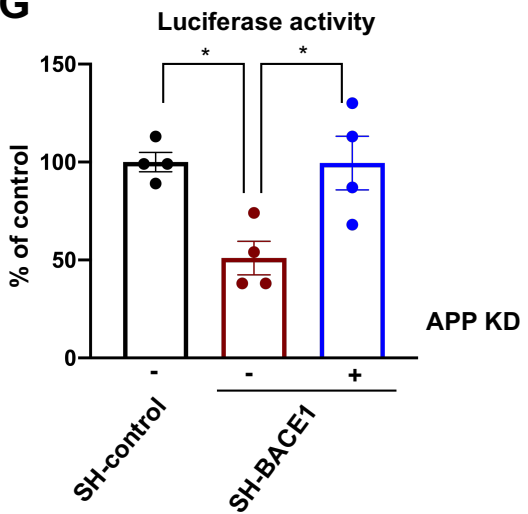

H Luciferase subunits
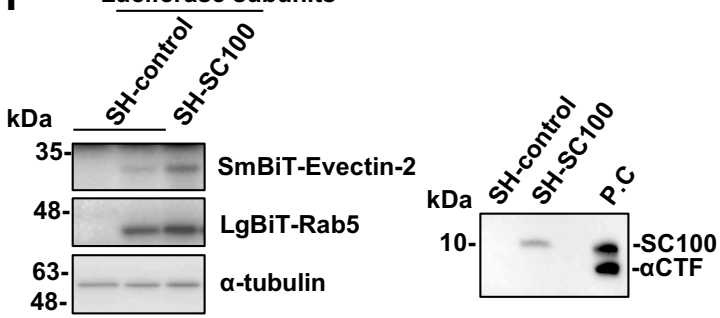

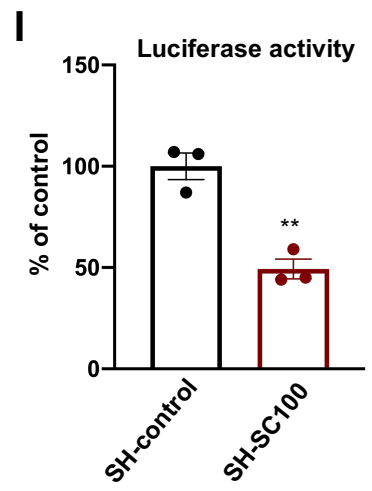




\section{Fig.4}

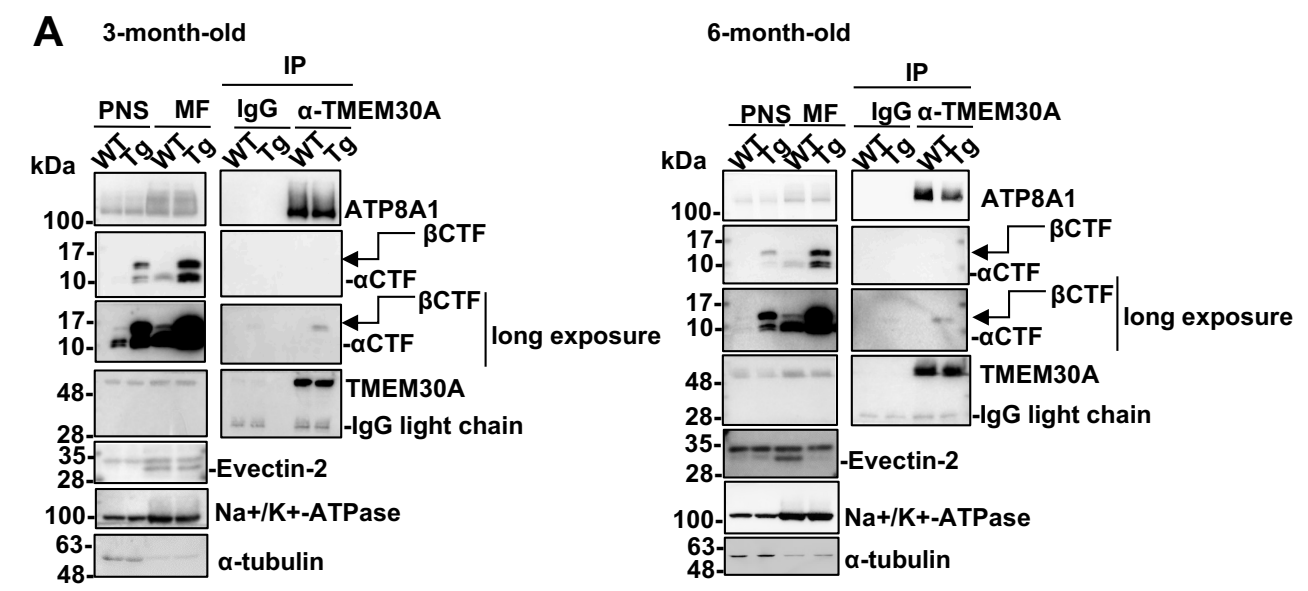

B TMEM30A / $\beta$ CTF complex formation
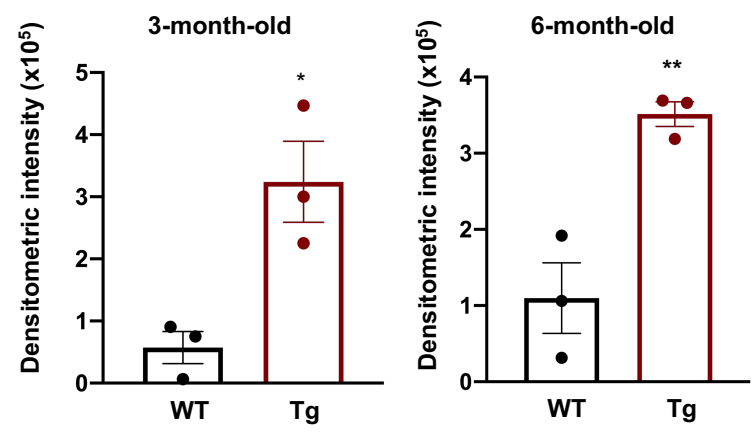

C

TMEM30A / ATP8A1 complex formation
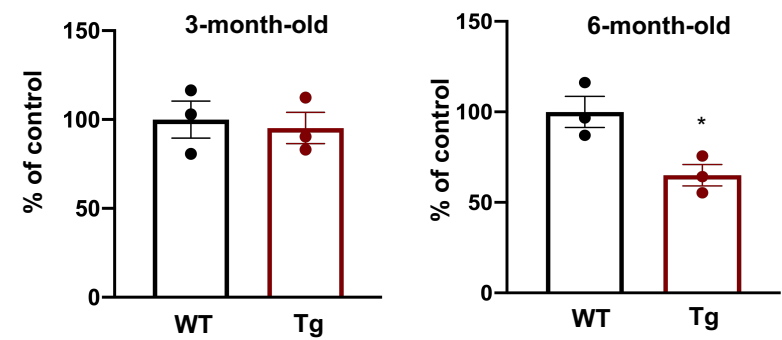

D

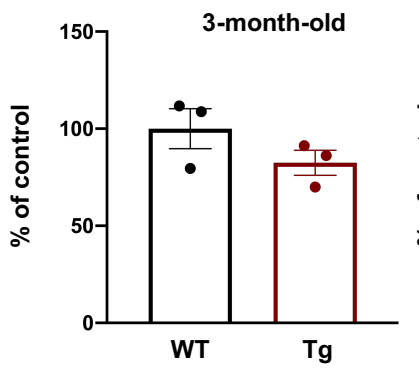


Fig.5

A

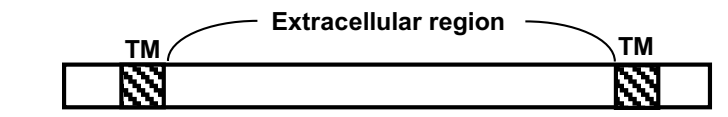

TMEM30A
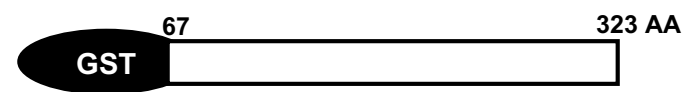

CST

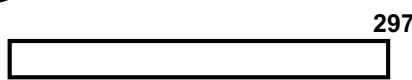

297

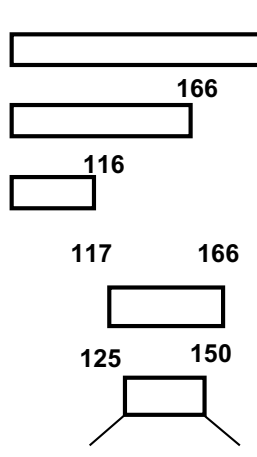

220

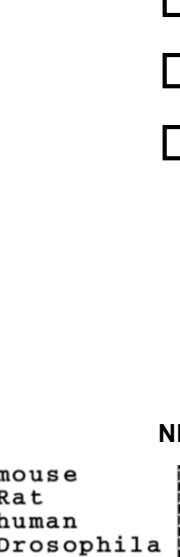

NFYQNHRRYVKSRDDSQLNGDPSAL

rosophila

C.elegans

\section{B}
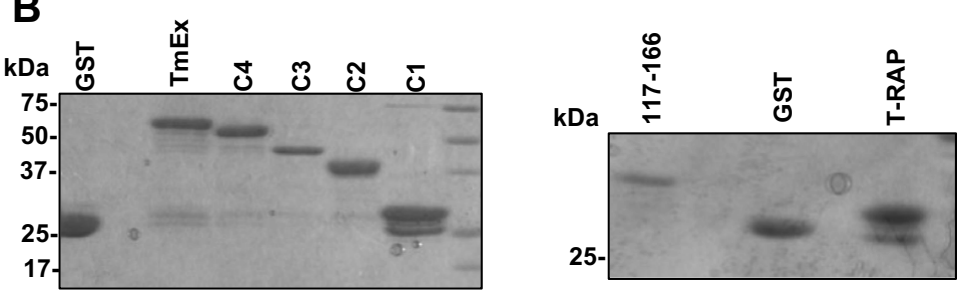

C

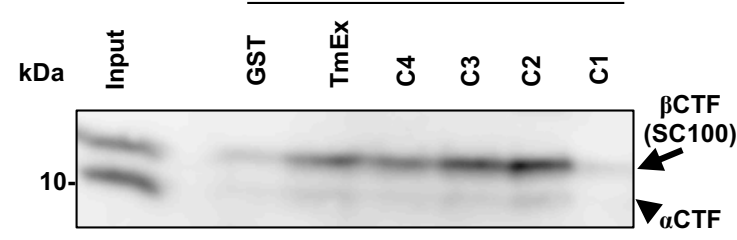

T-RAP(125-150)

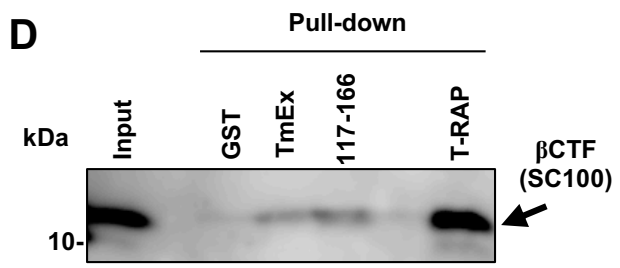


Fig.6

A

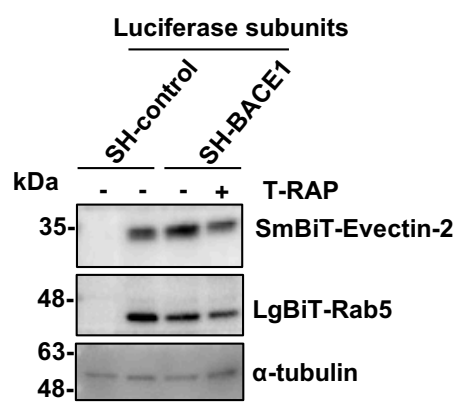

D

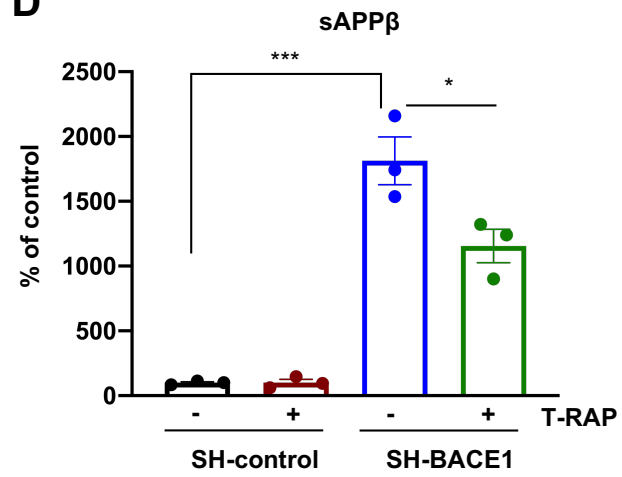

B

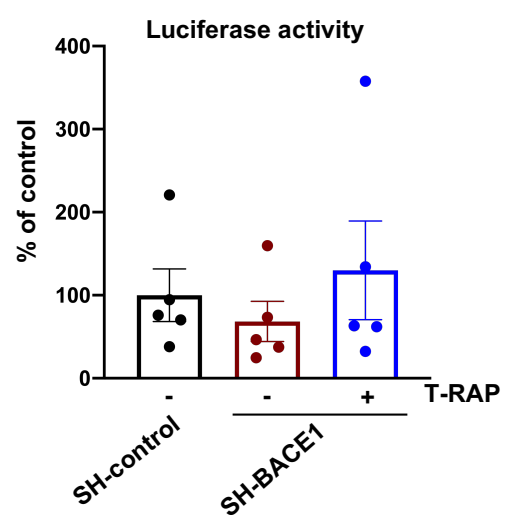

C

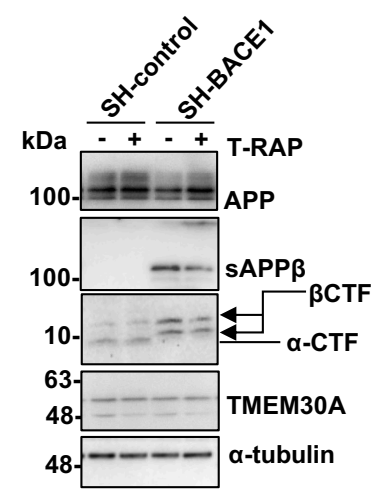

E

cos-control

-

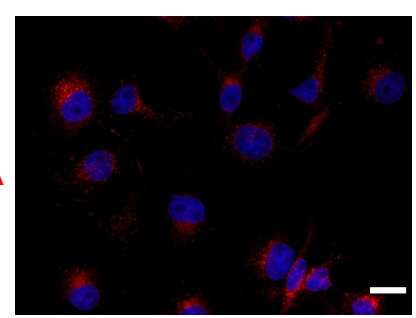

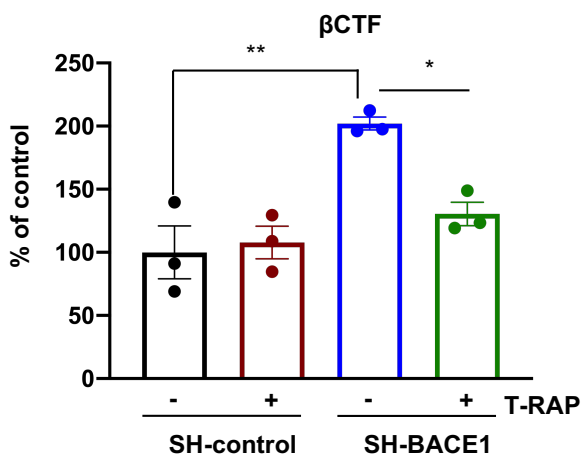

F

COS-BACE1

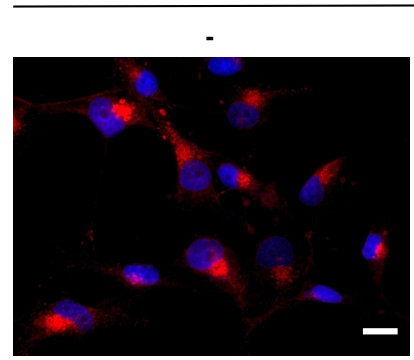

T-RAP

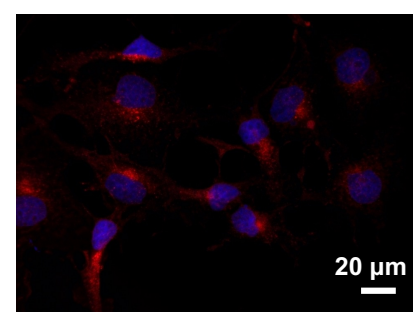

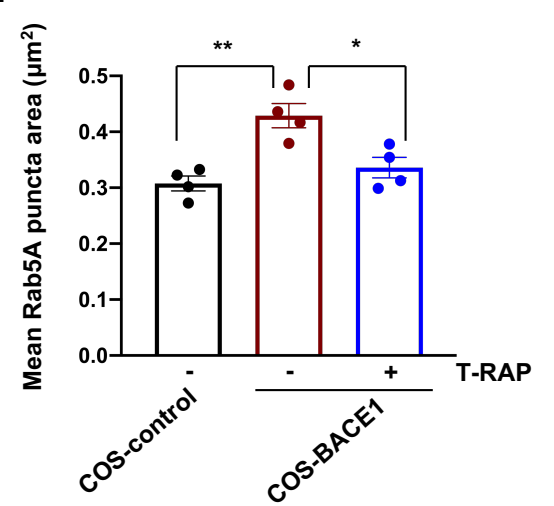


bioRxiv preprint doi: https://doi org/10.1101/2021 07.30.454423; this version posted August 29, 2021. The copyright holder for this preprint (which was not certified by peer review) is the author/funder, who has granted bioRxiv a license to display the preprint in perpetuity. It is made available under aCC-BY-NC-ND 4.0 International license.

Fig.7

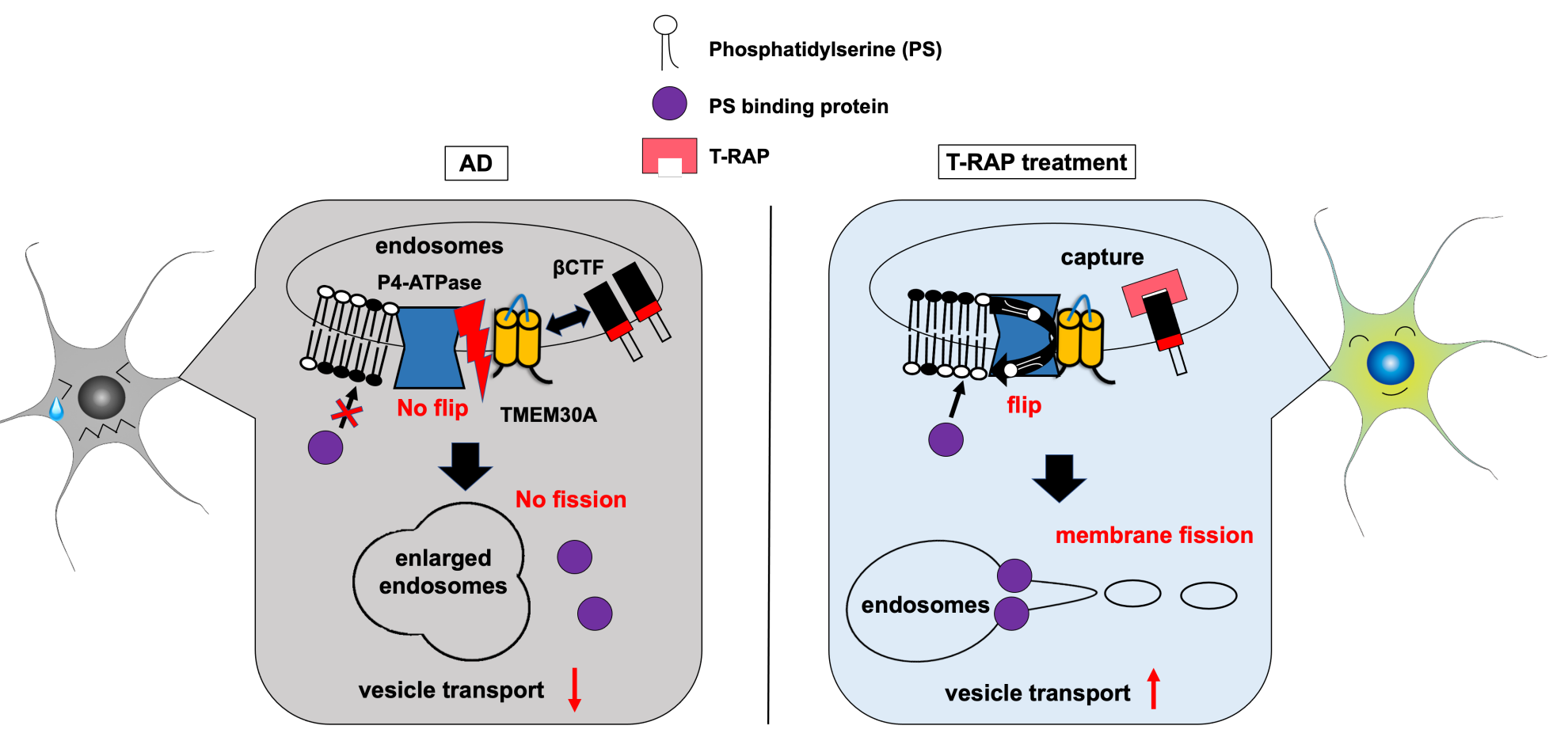

